\title{
Monseigneur Provencher et son clergé séculier
}

\section{David Roy}

Volume 37, 1970

La vie religieuse au Manitoba

URI : https://id.erudit.org/iderudit/1007272ar

DOI : https://doi.org/10.7202/1007272ar

Aller au sommaire du numéro

Éditeur(s)

Les Éditions Historia Ecclesiæ Catholicæ Canadensis Inc.

ISSN

0318-6172 (imprimé)

1927-7067 (numérique)

Découvrir la revue

Citer cet article

Roy, D. (1970). Monseigneur Provencher et son clergé séculier. Sessions d'étude - Société canadienne d'histoire de l'Église catholique, 37, 1-16.

https://doi.org/10.7202/1007272ar

Tous droits réservés @ C Les Éditions Historia Ecclesiæ Catholicæ Canadensis Inc., 1972
Ce document est protégé par la loi sur le droit d'auteur. L'utilisation des services d’Érudit (y compris la reproduction) est assujettie à sa politique d'utilisation que vous pouvez consulter en ligne.

https://apropos.erudit.org/fr/usagers/politique-dutilisation/ 


\section{Monseigneur Provencher et son clergé séculier}

\section{Un cent cinquantième anniversaire}

J'aimerais signaler une omission bien involontaire à la liste des anniversaires inscrite sur le programme. Il s'agit de la promotion à l'épiscopat de l'abbé Joseph-Norbert Provencher, il y a cent cinquante ans cette année.

En effet, le $1^{\text {er }}$ février 1820 , le pape Pie VII, à la demande de monseigneur Júseph-Octave Plessis, nommait l'abbé Provencher au titre " d'Évêque de Juliopolis sous l'Archevêché d'Ancyre, in partibus infidelium, ... en qualité d'Auxiliaire suffragant et de Vicaire Général de ce même Archevêque de Québec. » "... pour la région ouest de l'Amérique britannique du nord, qui s'étend de la Baie d'Hudson et de la Baie James, limitée à l'est par les Provinces du Haut et du Bas-Canada, et à l'ouest par les Montagnes Rocheuses ${ }^{1}$." Le même jour, l'abbé Jean-Jacques Lartigue était préconisé évêque de Telmesse pour le district épiscopal de Montréal.

L'abbé Provencher était à la rivière Rouge à ce moment-là en qualité de vicaire général de monseigneur Plessis. Il y était arrivé avec l'abbé Sévère Dumoulin le 16 juillet 1818. Il se préparait à retourner en Canada, après deux ans d'expérience, pour faire rapport sur l'état de la nouvelle mission. Il ne pouvait pas savoir que le souverain pontife venait de poser un geste qui devait consacrer définitivement l'établissement de l'Église dans ce vaste territoire du Nord-Ouest et de la Terre de Rupert. Cinquante ans plus tard, en 1870, ces territoires devaient passer à la Confédération canadienne pour faire partie intégrante du Canada.

C'est heureux, d'une manière, que l'on ait omis cet anniversaire. J'ai eu bien garde de le faire remarquer avant aujourd'hui, car j'aurais été privé d'une "introduction» qui me fit entrer de plain-pied dans mon sujet $^{2}$ :

"Monseigneur Provencher et son clergé séculier. "

1 Bulle de Pie VII, donnée à Rome le $1^{\text {er }}$ février 1820 , sous la signature du cardinal Consalvi. Copie, Rég. A., p. 39, AASB. La traduction du texte latin est donnée dans Les Cloches de Saint-Boniface, 1953, p. 180-181. Le traducteur met le mot * Canada s au lieu d'e Amérique Britannique du Nord `. J'ai consulté abondamment cette revue diocésaine; dorénavant, je la citerai simplement comme suit: Les Cloches.

2 J'éprouve toujours beaucoup de peine à introduire un sujet, et mes paroissiens trouvent que j'ai encore plus de misère $a ̀$ le conclure. 


\section{Comparaisons et rapprochements}

Si l'on me permettait d'être imaginatif, tout en respectant la vérité historique scrupuleusement documentée, j'établirais une espèce d'analogie entre monseigneur Provencher et ses prêtres, et le Christ et ses apôtres, car il y a, en effet, d'intéressants rapprochements à faire. On pourrait accepter un "sens accommodatiste" de la description des lieux, des conditions de vie, du comportement des personnes et de l'interprétation des faits. La tentation est grande, et j'avoue que j'y cède volontiers... et que j'y prends plaisir.

\section{a) Les lieux}

On pourrait d'abord établir une analogie (secundum quid idem... simpliciter diversa) entre le territoire de la rivière Rouge et la Palestine. $\mathrm{La}$ rivière Rouge serait notre Jourdain. Elle prend une de ses sources ${ }^{3}$ dans le lac Traverse au sud et se jette dans le lac Winnipeg au nord. Ce lac Traverse, en territoire américain, est fortement incrusté à l'intérieur de la hauteur des terres qui constitue ce que les américains appellent "The Continental Divide». C'est la grande division entre le versant du sud et le versant du nord du continent nord-américain. Au versant sud, les rivières de l'Amérique centrale se jettent dans le Mississippi pour se déverser ensuite dans le Golfe du Mexique. Au versant nord, les rivières se jettent dans la rivière Rouge ou le lac Winnipeg pour se déverser dans la baie d'Hudson. Avec une bonne dose d'imagination, ce lac Traverse, avec ses eaux boueuses et presque stagnantes, nous ferait penser à la mer Morte.

Le lac Winnipeg, au contraire, poissonneux, et très capricieux au moment de la tempête, véritable mer intérieure puisqu'il est un des plus grands lacs au Canada, se comparerait au lac de Tibériade ou à la mer de Galilée.

La baie d'Hudson se comparerait avantageusement avec la mer Méditerranée, et les rochers du "Bouclier canadien " offraient, au temps de monseigneur Provencher, des risques et des dangers comparables aux montagnes de la Palestine.

Nos plaines de l'Ouest, qui étaient d'immenses solitudes avant la colonisation, ressemblaient à d'immenses déserts ${ }^{4}$. Après les grands feux de prairie qui avaient détruit toute végétation et $\mathrm{y}$ avaient fait périr "la vache " qui s'y alimentait ${ }^{5}$, les gens se sentaient justifiés de l'appeler

3 J'apporte ici une carte préparée par le « Water Conservation Branch * du département des Ressources naturelles de la province du Manitoba, numérotée "Plan $n^{\circ}$ 10-1-1001 ", où l'on voit tous les systèmes des rivières qui se jettent dans la rivière Rouge avant de se déverser dans le lac Winnipeg.

4 Voir monseigneur TACHÉ, Esquisse sur le Nord-Ouest de l'Amérique, 1888, Montréal, $2^{\mathrm{e}}$ éd., 1901, p. 6.

5 Voir lettre Provencher-Panet $\left(\mathrm{M}^{\mathrm{gr}}\right.$ de Saldes), Saint-Boniface de la Rivière Rouge, 24 novembre 1818, BSHSB, III, p. 42. 
ainsi. Pour les traverser et se rendre au pied des montagnes Rocheuses, ça ne devait pas prendre beaucoup moins de «quarante jours et quarante nuits ${ }^{6}$ ".

Avec l'arrivée des missionnaires à la rivière Rouge, Saint-Boniface devint le centre de la chrétienté pour tout l'Ouest canadien et le NordOuest américain, comme Jérusalem, la Ville sainte, était depuis l'antiquité le centre du culte au seul vrai Dieu.

Bien plus, la première cathédrale en pierre de monseigneur Provencher, projetée dès $1829^{7}$, construite au cours des années 1834 à 1839, et ouverte au culte le 27 octobre $1839^{8}$, était comme le Temple de Salomon, le monument le plus majestueux et le plus imposant de toutes ces contrées ${ }^{9}$. Elle fut détruite par le feu le 14 décembre 1860 . Et notre dernière basilique-cathédrale, détruite par le feu le 22 juillet 1968 et encore à l'état de ruine historique, ne nous fait-elle pas penser au majestueux temple de Jérusalem, qui excitait l'admiration des Apôtres, et détruit de fond en comble lui aussi ?

Vous trouverez ces comparaisons capricieuses, peut-être, pour situer, les lieux où se déroulèrent les activités apostoliques de monseigneur Provencher et ses prêtres. Pour être plus vrais, ces rapprochements doivent tenir compte du fait que, contrairement au Jourdain, notre rivière Rouge coule du sud au nord, et que le territoire en question est presque à l'étendue d'un continent, tandis que la Palestine n'est qu'une toute petite particule du Moyen-Orient.

Serait-il opportun de rappeler brièvement les grands fléaux qui s'abattirent sur la mission de la rivière Rouge, et qui, eux aussi, pourraient avoir une résonance quelque peu biblique? L'épidémie de sauterelles de 1818 à 1820 et l'état de grande misère sinon de famine qui s'ensuivit rappellent une des plaies d'Égypte. Les deux grandes inonda-

6 Monsieur l'abbé Thibault se rendit, à dos de cheval, à Edmonton ou Fort des Prairies en 1842. Il partit de Saint-Boniface le 20 avril 1842 * allant à petit train, ses chevaux étant faibles à cause du mauvais pacage de cette saison $*$. Provencher-Signay, Saint-Boniface de la Rivière Rouge, 30 juin 1842, BSHSB, III, p. 200.

7 Lettre Provencher-Panet, Saint-Boniface, 6 juin 1829, BSHSB, III, p. 126. - Le Gouverneur Simpson... de son propre mouvement m'a offert cent louis si je voulais bâtir une église en pierre. " Lettre Provencher-Panet, Saint-Boniface de la Rivière Rouge, le $1^{\text {er }}$ juillet 1829, BSHSB, III, p. 130. M. Simpson doit parler pour faire circuler parmi les catholiques de Londres une souscription pour bâtir l'église de St-Boniface.» L'archevêque de Québec, dans une lettre circulaire au clergé en 1831, encouragea une semblable souscription auprès des prêtres et des fidèles du Québec. L'Association de la Propagation de la Foi de Lyon a pourvu au reste de la dépense. Voir monseigneur Provencher, Notice sur la Rivière Rouge , in Les Cloches, 1927, p. 117.

8 Lettre Provencher-Blanchet, Saint-Boniface de la Rivière Rouge, 26 décembre $1839,3^{\mathrm{e}}$ page, archives de l'archevêché de Portland, Oregon (copie au Xerox).

9 Cf. Professor Henry Yule Hind, in Red River, Assiniboine and Saskatchewan expedition of 1857 ». 
tions de 1826 et de 1852 , qui ne laissèrent partout que des décombres, devaient paraître comme un mini-déluge. Par ailleurs, les froids intenses de l'hiver devaient faire oublier toute ressemblance avec la Terre Sainte. Malgré tout cela, en lisant leur bréviaire, monseigneur Provencher et ses missionnaires pouvaient s'appliquer les mots du psalmiste: "Per ignem et aquam transivi. »

\section{b) Les missionnaires du clergé séculier}

Si l'on observe maintenant les personnes des missionnaires, on pourrait dire que Saint-Boniface a eu son Seigneur et ses douze apôtres (excepté Judas).

Monseigneur Provencher a vécu trente-cinq ans comme missionnaire à la rivière Rouge. Si l'on comptait ses années d'épiscopat à partir de 1820, jour où il fut préconisé évêque, ça lui donnerait, lui aussi trentetrois ans de vie épiscopale avant sa mort, survenue à Saint-Boniface le 6 juin $1853^{10}$.

Sous l'épiscopat de monseigneur Provencher, Saint-Boniface a eu ses douze apôtres. En effet, douze prêtres sont venus œuvrer dans la « vigne du Seigneur » de 1818 à 1853. On pourrait même en ajouter un treizième, un autre Paul, apôtre des nations, dans la personne de l'abbé Albert Lacombe, sans cependant lui faire partager l'épithète d'\& avorton ». En effet, ce grand missionnaire était encore du clergé séculier à la mort de monseigneur Provencher, bien qu'il ait fait connaître son ardent désir de joindre la congrégation des Oblats de Marie-Immaculée ${ }^{11}$.

Voici donc la liste de ces douze prêtres. C'est monseigneur Taché qui la donne ${ }^{12}$.

Sévère Dumoulin

Thomas Picard-Destroismaisons

Jean Harper

François Boucher

Georges-Antoine Belcourt

Charles-Edouard Poiré

Jean-Baptiste Thibault

Modeste Demers (évêque de Vancouver)

Joseph-Arsène Mayrand

Jean-Edouard Darveau

Louis-François Laflèche

Joseph Bourassa

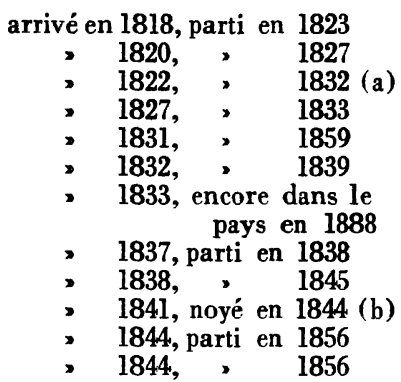

10 Voir la mort et les funérailles de monseigneur Provencher (d'après le journal des révérendes Sœurs Grises), in Les Cloches, 1922, p. 67 sq.

11 Le Père Lacombe, Mémoires et souvenirs, imprimé au Devoir, Montréal, 1916, voir p. 54 à 56 .

12 Monseigneur TACHÉ, Vingt années de Missions, Montréal, nouvelle édition, 1888 , p. 21, note 1. Je cite la liste telle qu'elle a été publiée, et j'ajoute: a) Jean Harper, parti le 22 juillet 1831: cf. BSHSB, III, p. 133; b) Monseigneur Provencher et monseigneur Taché crurent que l'abbé Darveau se fût noyé. Ce ne fut que longtemps après que le père Camper, o.m.i., apprit des sauvages eux-mêmes qu'il avait été massacré. Cf. Les Cloches, 1912, p. 186, et 1914, p. 185. 
Il conviendrait d'ajouter ici les noms de deux ecclésiastiques qui ont fait partie de l'équipe missionnaire des débuts et qui n'ont pas persévéré dans l'état ecclésiastique. Guillaume Edge accompagna les abbés Provencher et Dumoulin en 1818. Il fit l'école à Pembina avec l'abbé Dumoulin, et retourna dans l'Est le 11 août 1820 avec l'abbé Provencher ${ }^{13}$. Joseph Sauvé arriva à la rivière Rouge avec l'abbé Picard-Destroismaisons le 12 août 1820. Lui aussi enseigna à l'école de Pembina. Il n'osa pas avancer aux ordres et demanda de retourner à Montréal. Il partit le 12 août $1822^{14}$.

$\mathrm{Au}$ risque de faire éclater tous les rapprochements que je viens de faire, serait-ce violenter la vérité historique que d'ajouter le nom de l'abbé François-Norbert Blanchet à la liste des noms déjà mentionné ? Je ne le crois pas.

L'abbẻ Blanchet était curé des Cèdres, près de Montréal. Monseigneur Provencher était de retour de son voyage à Rome en 1836 et il passait l'hiver en Canada en attendant les canots du printemps 1837 qui devaient le ramener à Saint-Boniface. Il s'occupa à trouver des missionnaires pour la Colombie, ou territoire d'Oregon comme on vint à l'appeler. Il s'adressa à l'abbé Blanchet pour lui offrir la mission de la Colombie et en fit part à l'archevêque de Québec ${ }^{15}$.

Malgré tous les contretemps et les déboires survenus, la mission fut une réussite. L'abbé Blanchet quitta Montréal le 3 mai 1838 et arriva à la rivière Rouge le 5 juin suivant. Monseigneur Signay, archevêque de Québec, l'avait fait son grand vicaire, avec la clause "Cum dependentia ab Ill'mo ac Rev'mo DD J.N. Provencher ${ }^{16}$ ». Cette clause, comme le faisait remarquer monseigneur Signay dans ses instructions aux missionnaires de la Colombie, était en conformité avec l'Indult du 28 février 1836, accordé par le pape Grégoire XVI, qui étendait la juridiction de monseigneur Provencher sur tout le territoire à l'ouest des montagnes Rocheuses, de la Californie à l'Alaska, « jusqu'à ce qu'il soit statué autrement par le Saint-Siège ${ }^{17}$ ».

Jusqu'au jour où monseigneur Blanchet fut consacré évêque, à Montréal, le 25 juillet 1845, comme vicaire apostolique de l'Oregon, monseigneur Provencher ne le considérait pas comme séparé de lui. Il sollicitait des octrois plus considérables de la Propagation de la Foi de Lyon, et les missions de la rivière Rouge et de la Colombie s'approvisionnaient $\grave{a}$ la même bourse ${ }^{18}$.

13 Cf. BSHSB, III, p. 13, 17, 35, 39, 42, 45, 57, et Les Cloches, 1927, p. 180.

14 Cf. Provencher, * Notice sur la Rivière Rouge ", in Les Cloches, 1927, p. 180, et Lettres, in BSHSB, III, p. 56, 61, 63, 73, 75, 77, 80.

15 Lettre Provencher-Signay, Nicolet, 17 novembre 1836, BSHSB, III, p. 156.

16 Lettre Signay-Provencher, Québec, 19 avril 1838, AAQR, L. 18:279.

17 Indult, 28 février 1836: AAQ, Rég. Ind., et Les Cloches, 1919, p. 50.

18 Lettre Provencher-Blanchet, Saint-Boniface, 20 juin 1843, archives de l'archevêché de Portland. 


\section{c) Les Oblats}

Je devrais me limiter aux seules personnes dont j'ai donné les noms, puisque mon sujet est "Monseigneur Provencher et son Clergé séculier ». Je ferai pourtant ici une exception pour mentionner aussi les noms des prêtres de la congrégation des Oblats de Marie-Immaculée qui ont aussi servi sous le premier évêque de Saint-Boniface. Ce sont les révérends pères dont les noms suivent:

Pierre Aubert, arrivé ici le 25 août 1845

Alexandre-Antonin Taché, arrivé ici le 25 août 1845 (futur évêque)

François-Xavier Bermond, arrivé ici en 1846

Henri Faraud, arrivé ici en 1846 (futur évêque)

Augustin Maisonneuve, arrivé ici le 15 septembre 1848

Jean Tissot, arrivé ici le 15 septembre 1848

Henri Grolier, arrivé ici avec monseigneur Taché et l'abbé Lacombe. le 27 juin 1852

René Rémas, arrivé le 11 septembre 1852, parti en juillet 1853

D. Végreville, arrivé le 12 septembre 1852. parti le 8 juin 185319

Si l'on a pu dire que le diocèse de Saint-Boniface était " tombé aux Oblats " à la mort de monseigneur Provencher, ce n'est certainement pas pour signifier une décadence, loin de là. Monseigneur Provencher, qui avait appelé de toutes ses forces les "Fils de Mazenod ", voyait en eux le seul gage de survivance et de progrès pour ses missions. Il se félicitait même d'avoir obtenu monseigneur Taché comme coadjuteur. par un véritable truchement providentiel, alors qu'il avait été à peu près décidé de retirer de la mission de la rivière Rouge ces braves missionnaires ${ }^{20}$.

C'est donc toute cette équipe de missionnaires qu'il convient d'honorer, si ce n'est que par une mention passagère, en reconnaissant le grand essor missionnaire dont ils ont été les artisans.

Quand on fait un rapprochement ou que l'on établit une comparaison, on force souvent la note pour signaler les ressemblances possibles. Je l'ai fait, mais en indiquant qu'il ne faudrait pas s'attacher " mordicus" au chiffre douze en parlant du clergé séculier de monseigneur Provencher. De fait. j'en ai nommé seize, en ajoutant. à la liste de monseigneur Taché, les noms de Blanchet et Lacombe, et des deux ecclésiastiques Edge et Sauvé. Si l'on ajoute encore à ces seize premiers noms ceux des neuf pères oblats mentionnés, on arrive au total de vingt-cinq collaborateurs venus à la rivière Rouge au cours des trente-cinq années de vie missionnaire de monseigneur Provencher. Ce serait un nombre

19 Régistre II, Actes Episcopaux, à la fin, AASB.

20 Voir lettre Provencher-Mazenod, Saint-Boniface, 29 novembre 1849, in Les Cloches, 1921, p. 154. Cette lettre, l'évêque de Marseille ne la reçut qu'après la nomination de monseigneur Taché. Cf. Provencher-Turgeon, 21 juillet 1851. BSHSB, III, p. 280. 
impressionnant si ces vingt-cinq collaborateurs s'étaient trouvés ensemble à la rivière Rouge.

\section{d) Collaborateurs peu nombreux à la fois}

Ce qui est moins impressionnant et qui est pourtant vrai, c'est que, du début et jusqu'à l'année 1844, soit les vingt-six premières années, monseigneur Provencher n'eut jamais plus de quatre prêtres à la fois avec lui ${ }^{21}$.

Quand Saint-Boniface fut érigé en vicariat apostolique le 16 avril 1844, le nombre de prêtres fut porté à cinq au cours de l'année, soit: Messieurs Belcourt, Thibault, Mayrand, Laflèche et Bourassa ${ }^{22}$. Ce nombre aurait pu être six, si l'abbé Darveau n'était pas mort, assassiné nar les sauvages, le 4 juin 1844. Monceigneur Provencher, qui arriva de son voyage d'Europe le 31 mai 1844, ne devait apprendre la triste nouvelle que le 14 juillet suivant ${ }^{23}$.

Ces changements continuels laissaient souvent l'évêque missionnaire dans une situation embarrassante et pénible, comme il l'écrivait aux abbés Blanchet et Demers. Après le départ de ceux-ci pour la Colombie, l'abbé Poiré était retourné en Canada, et l'abbé Belcourt était parti lui aussi pour revenir l'année suivante. Monseigneur Provencher écrivait:

En voilà deux de partis dans la même année et un de changé. Je n'y ai pas gagné. M. Thibault, parti d'ici la larme à l'œil pour la mission sauvage, a été bien content de voir partir $M^{\mathrm{r}}$ Poiré pour prendre sa place, ce qui le rapprochait un peu. ...Je reste avec M. Mayrand qui est décidé à ne pas essayer d'apprendre la langue sauvage. Me voilà bientôt réduit à rien pour ces pauvres infidèles 24 .

\section{e) Leur jeune âge}

Une autre caractéristique des collaborateurs de monseigneur Provencher fut leur jeune âge. Il faut en tenir compte vu la multiplicité des responsabilités missionnaires qu'ils étaient appelés à assumer dès leur arrivée au pays. Harper avait 20 ans, Poiré 21, Thibault 22, Destroismaisons et Boucher 24, Dumoulin, Darveau et Lacombe 25, Laflèche 26, Demers et Bourassa 27, Belcourt 28. Monseigneur Provencher lui-même n'avait que 31 ans quand il arriva à la rivière Rouge, tandis que

21 Joseph-Etienne Champagne, o.m.i., Les Missions de lOuest canadien, Ottawa, 1949, p. 66.

22 Monseigneur TACHÉ, * Rapport de 1888 , in Les Cloches, 1915, p. 131.

23 Cf. lettre Provencher-Bourget, Saint-Boniface, 26 juin 1844; Les Cloches, 1919, p. 243; Provencher-Signay, Saint-Boniface, 29 juillet 1844, BSHSB, III, p. 236; Provencher-Bourget, Saint-Boniface, 30 juillet 1844, in Les Cloches, 1919, p. 263 .

24 Lettre Provencher-Blanchet, Saint-Boniface de la Rivière Rouge, 13 décembre 1839. Citée d'une copie au Xerox de l'original conservé aux archives de l'archevêché de Portland, Oregon. 
Blanchet était déjà d'âge mûr - il avait 43 ans - quand il arriva à la rivière Rouge, en route pour la Colombie.

Monseigneur Provencher, qui vieillissait tous les ans comme tout le monde, aurait-il ressenti l'écart d'âge entre lui et ses jeunes compagnons? C'est vraisemblable, mais nullement prouvé. Il écrivait aux évêques de Québec à peu près en ces termes: "Que voulez-vous que l'on fasse avec des jeunes pris sur les bancs du séminaire... qui retournent dès qu'ils sont capables de se montrer ou dès qu'ils savent assez la langue des sauvages pour être utiles. "Ces remarques visent beaucoup plus à faire voir l'inconvénient qu'il y avait de remplacer des prêtres ayant acquis l'expérience de la vie missionnaire par des candidats inexpérimentés, que de critiquer la jeunesse de ceux qui généreusement prêtaient leurs services. Monseigneur était ni grognard ni bougonneux !

\section{Une légende}

Dans ce contexte, je crois que la légende qui s'est bâtie autour de l'arrivée du frère Taché à Saint-Boniface, le 25 août 1845, est peu vraisemblable et beaucoup plus folklorique que vraie. Laissons d'abord le témoin principal nous en parler.

En 1888, monseigneur Taché lui-même écrivait:

Le lendemain, fête de saint Louis, notre canot s'arrêtait devant la cathédrale de Saint-Boniface. Monseigneur Provencher descendit au rivage recevoir les deux missionnaires que la Congrégation avait choisis pour aller annoncer au pieux prélat que désormais notre chère famille dirait avec lui: (

Le dimanche suivant, $1^{\text {er }}$ septembre, le Frère Taché, qui pendant le voyage avait atteint sa vingt-deuxième année, fut ordonné diacre par $\mathbf{M}^{\mathbf{E}}$ Provencher. Le zélé pontife profita de la circonstance pour dire à son peuple le bonheur que lui procurait l'arrivée des missionnaires religieux Oblats. Empruntant les paroles du vieillard Siméon, il dit à Dieu: - Nunc dimittis servum tuum Domine... quia viderunt oculi mei salutare tuum, quod parasti ante faciem omnium populorum. " A la pensée que la lumière se répandrait parmi toutes les nations de son immense vicariat, une profonde émotion s'empara du çeur de l'apôtre de la Rivière Rouge; les sanglots étouffèrent sa voix, et les larmes de tout son peuple se joignirent aux siennes 25 .

Monseigneur Taché ajoute qu'il fut ordonné prêtre le 12 octobre 1845 , et qu'il fit son oblation aux mains du père Aubert. C'était la première profession religieuse faite dans l'Ouest, et le religieux qui la fit était " un des arrière-neveux " de la Vérendrye, le grand découvreur ${ }^{26}$. Évidemment, le jeune père Taché n'a retenu que la joie de l'évêque et sa profonde émotion.

25 Monseigneur TACHÉ, Vingt années de Missions, Montréal, 1888, p. 26.

26 Monseigneur TACHÉ, loc. cit. 
L'abbé Georges Dugas qui écrivait la vie de monseigneur Provencher, l'année suivant la publication du livre de monseigneur Taché, soit en 1889 , commence discrètement la légende.

A l'arrivée du jeune sous-diacre, portant encore les traits d'un adolescent, $\mathrm{M}^{\mathrm{gr}}$ Provencher sembla éprouver une espèce de désappointement; il attendait des ouvriers prêts à se mettre à l'ouvrage; il eût préféré voir arriver un prêtre; cependant il ne fut pas longtemps sans s'apercevoir qu'il possédait, dans le Frère Taché, un sujet distingué, doué de rares talents et de qualités admirables. Ainsi quelques semaines plus tard, il écrivait ces paroles à l'évêque de Québec: - Des Taché et des Laflèche, vous pouvez m'en envoyer sans crainte ${ }^{27}$. ,

J'ai cherché dans les lettres de monseigneur Provencher pour situer cette citation dans son contexte. Voici ce que j'ai trouvé dans une lettre du 14 juin 1847, donc deux ans après et non pas * quelques semaines plus tard $»$.

J'aimerais à avoir quelques prêtres canadiens, mais il faudra du choix: si vous aviez encore des Laflèche ou des Taché ! La congrégation des Oblats a acquis quelques canadiens qui ne déshonoreront pas le clergé, j'espère, et qui pourront se montrer avec leurs confrères d'outremer. Tant mieux, il nous en reviendra peut-être quelques-uns; malheureusement il n'y a pas de vocations dans votre diocèse. Je n'aime pas cette nullité 28 .

Le grand maître de la légende est sans aucun doute le vénérable Dom Benoît qui écrivit la vie de monseigneur Taché en 1904, quand il rapporte ce qui est supposé être arrivé. Je le cite textuellement et vous serez en mesure de comparer. Vraiment, l'heureux événement tourne au tragique !

Quelques habitants avaient vu passer le canot et étaient courus avertir $\mathbf{M}^{\mathrm{gr}}$ Provencher. Celui-ci était au rivage avec $\mathrm{M}$. Mayrand et les Sœurs Grises pour recevoir les deux missionnaires... etc. [II cite $M^{\mathrm{gr}}$ Taché tel que ci-haut.]

Les deux missionnaires se prosternèrent devant le vénérable évêque, qui les bénit et les releva [c'est vraisemblable]. Le $P$. Aubert, après avoir donné son nom, donna celui de son compagnon, Frère Taché, sous-diacre [rien de plus normal]. . Sous-diacre!, se récria vivement $\mathrm{M}^{\mathrm{gr}}$ Provencher, se rappelant un sous-diacre qui lui avait été envoyé moins pour l'aider, que pour débarrasser ses supérieurs ecclésiastiques. - Sous-diacre! Mais ce sont des prêtres qu'il nous faut !. . Je le sais, Monseigneur, reprit le P. Aubert; mais il n'avait pas l'âge canonique pour être ordonné diacre lorsqu'il est parti de Montréal; mais comme le voyage a été long, il a maintenant cet âge: Votre Grandeur pourra l'ordonner quand elle le voudra. : A la bonne heure! répondit le prélat: il nous est venu des ecclésiastiques qui n'étaient pas capables d'être prêtres, qui même ont donné du scandale! je craignais déjà... Ici il faut du premier choix. - Un instant après, jetant un regard furtif sur le sous-diacre dont la physionomie exprimait un âge moindre encore qu'il n'était, l'évêque s'échappa à dire: ‘ On m'envoie des enfants! Ce sont des hommes qu'il nous faut. " [Quelle jérémiade !] $\mathrm{M}^{\mathrm{gr}}$ Provencher

27 Georges Dugas, Monseigneur Provencher, 1889, p. 237.

28 Lettre à monseigneur P.-F. Turgeon, évêque de Sidyme à Québec, SaintBoniface de la Rivière Rouge, 14 juin 1847, BSHSB, III, p. 261. 
ne tardera pas à revenir de ses premières impressions. Il écrira bientôt à l'évêque de Québec: * Des Taché et des Laflèche, vous pouvez m'en envoyer sans crainte 29 , [Il cite ici mot à mot Dugas sans l'indiquer.]

Dom Benoît donne comme référence à toutes ces trouvailles monseigneur Taché lui-même dans Vingt années de Missions..., p. 26, texte que j'ai cité moi-même en entier. C'est fantastique! Le fait, tel qu'il le rapporte, prend l'allure d'un véritable incident, d'une " affaire " telle que les journalistes aimeraient la rapporter. Rien dans le tempéramment de monseigneur Provencher, ni dans ses lettres, ne permet de la supposer. Ni monseigneur Provencher, ni l'abbé Dugas ne parlent de "sous-diacres scandaleux" dont l'évêque de Québec aurait voulu se débarrasser sur le pauvre Provencher. Il y a bien une mention très vague, à un endroit, où monseigneur Provencher, récemment consacré, et en route pour la rivière Rouge, rapporte une lettre de M. Dumoulin:

M. Dumoulin se plaint que la mission a souffert des scandales cet hiver; la dispersion de son troupeau en a été la cause en grande partie 30 .

C'est une pure fabrication d'une imagination trop fertile.

Dom Benoît faisait-il allusion à M. Guillaume Edge, compagnon des abbés Provencher et Dumoulin à la rivière Rouge, en 1818 ? Peutêtre. Mais Edge n'était pas sous-diacre, il n'était plus " jeune" puisque à vingt-six ans il avait un an de plus que Dumoulin, et il n'y a aucune indication chez lui de conduite scandaleuse. L'abbé Provencher le ramena avec lui à Montréal en 1820; il allait avoir vingt-neuf ans, deux mois après son arrivée.

Le pire que l'abbé Provencher ait pu dire de lui se résume dans les trois passages suivants de ses lettres que je cite:

M. Edge n'est pas du tout ce qu'il faut. Monseigneur [Plessis] m'écrit qu'il n'a pas trop de répugnance à rester ici et me conseille de descendre seul. Ce plan me plairait si ce jeune homme était propre à cet œuvre. Il resterait volontiers: mais il descendra très certainement. M. Roux avait dit, avant notre départ de Montréal, que ce jeune Monsieur n'était pas ce qu'il fallait. Je suis fâché qu'il ne se soit pas trompé ${ }^{31}$.

Monsieur Edge est descendu avec moi après avoir été indécis si je le ferais descendre ou non. Je prie votre Grandeur de ne rien régler de sa destination future avant mon arrivée à Québec, mais de le laisser voir sa famille à Montréal jusqu'à ce temps ${ }^{32}$.

Presque un an après, l'abbé Provencher, devenu curé de Yamachiche, mentionne l'abbé Edge de la façon suivante:

29 Dom Benoît, Vie de $M^{g r}$ Taché, Montréal, vol. I, 1904, p. 86-87.

30 Provencher-Plessis, Fort William, 8 juillet 1822, BSHSB, III, p. 73.

31 Provencher-Panet, Saint-Boniface, 24 novembre 1819, BSHSB, III, p. 42.

32 Provencher-Plessis, Montréal, 17 septembre 1820, BSHSB, III, p. 45. Estce erreur d'édition? Monseigneur Provencher dit qu'il partit de la rivière Rouge - le 16 août s et qu'il prit « deux mois » pour faire le voyage à Montréal. Il faudrait écrire octobre au lieu de septembre. 
L'abbé Edge est-il revenu au séminaire ou s'est-il décidé à quelqu'autre état ? Je crois qu'il quittera la soutane difficilement. C'est un homme qui voulait être prêtre à quelque prix que ce fut sans se mettre en peine des antécédents et on peut ajouter des conséquences. J'ai cherché plusieurs fois à le dégoûter de cet état sans qu'il y ait mordu 33 .

La dernière fois que monseigneur Provencher fait mention de $\mathrm{M}$. Edge, c'est dans sa Notice sur la Rivière Rouge, publiée en collaboration en $1843^{34}$. On y trouve un simple rappel de son passage à la rivière Rouge, avec courte identification historique, sans plus:

M. Jh. Sauvez, ecclésiastique, élève de Nicolet, né le 8 décembre 1784 à Vaudreuil, fut son [de Destroismaisons] compagnon de voyage; il devait remplacer M. William Edge, autre ecclésiastique, monté à la Rivière Rouge en 1818; celui-ci était né à Montréal le 1 décembre 1792 et avait étudié au collège de la même ville; il devait descendre avec M. Provencher. Ces deux jeunes messieurs n'ont pas persévéré dans l'état ecclésiastique ${ }^{35}$.

Une fois rendu à Montréal, il paraît qu'il s'est rangé du côté de ceux qui s'opposaient à la formation du district épiscopal de la rivière Rouge :

Des missionnaires qui étaient de l'Ouest, Edge par exemple, soutenaient que les arrangements projetés étaient prématurés. De toutes parts, on envisageait comme impossible de pourvoir à un tel établissement. C'est pourquoi Lartigue conseilla à $\mathbf{M}^{\mathrm{gr}}$ Plessis de garder quelques années dans ses tiroirs les lettres apostoliques de Provencher ${ }^{36}$.

Le père A. G. Morice, o.m.i., continue la légende dans son livre sur l'Histoire de l'Église Catholique dans l'Ouest Canadien, publié en 1912. Il $n$ 'indique pas ses sources bien qu'on les reconnaisse:

Quand l'évêque aperçut les religieux qu'on lui envoyait, sa première impression ressembla quelque peu à un désappointement. Au lieu d'une petite bande de prêtres tout prêts à commencer leurs travaux apostoliques, il n'en voyait qu'un, le R.P. Pierre Aubert, qui était accompagné d'un jeune homme à la mine presqu'enfantine, qui lui paraissait à peine sorti de l'adolescence.

- Quoi ! s'écria-t-il à cette vue, j’avais demandé des hommes, et voilà qu'on m'envoie un enfant !

33 Provencher-Plessis, Yamachiche, $1^{\text {er }}$ septembre 1821, BSHSB, III, p. 57.

34 Cette notice fut écrite vraisemblablement en collaboration avec l'abbé Cazeault. "Il devient de plus en plus urgent d'écrire une relation à Lyon sur mes missions. Je serai obligé d'aller à Québec travailler avec M. Cazeault, mais il faudrait qu'il n'eut que cela à faire, ou l'envoyer à Nicolet avec tous les documents nécessaires pour composer une relation un peu intéressante. Ce serait le parti le plus sûr pour que je puisse en tirer de l'aide. Si la proposition plaisait à votre Grandeur et au brave secrétaire, il pourrait me joindre à la fin de la retraite " (voir Lettre Provencher à Signay, Montréal, 19 octobre 1843, BSHSB, III, p. 216).

35 Provencher, "Notice sur la Rivière Rouge ,, in Les Cloches, 1927, p. 180. C'est la seule fois qu'il lui donne le nom de "William, au lieu de a Guillaume *. Sans doute à cause de son ascendance anglaise.

36 Lucien Lemieux, Etablissement de la première province ecclésiastique au Canada, 1783-1844, Montréal, Fides, 1968, p. 133. 
Il ne tarda pas à constater que cet « enfant > n'était pas ordinaire, et quelques semaines plus tard il en demandait d'autres de cette espèce ${ }^{37}$.

Ensuite Morice parle de Taché, disant: "Il était du côté de sa mère un descendant directe du découvreur du pays, le grand Lavérendrye.» Monseigneur Taché se disait simplement * un des arrièresneveux ", et le père Antonio Champagne nous montre bien comment finit la lignée directe du grand découvreur en 1825, par la mort du dernier de ses fils ${ }^{38}$.

Faut-il mentionner que Donatien Frémont ${ }^{39}$ offre une version de la légende qui se rapproche de Dom Benoît, sans références, tandis que le R. P. Joseph.Étienne Champagne ${ }^{40}$ suit plutôt Morice et Dugas, mais au moins il y réfère honnêtement.

Quoi qu'on en dise, la légende est lancée et répétée d'auteur en auteur. On citera les noms de Dom Benoît et de Morice pour montrer que monseigneur Provencher était sujet à des accès de mauvaise humeur, qu'il s'efforçait bien de réparer par la suite. Le portrait du jeune père Taché est toujours vraiment juvénile. Le proverbe italien, en fin de compte, demeurera le critère de la vraisemblance des faits: "Se non e vero, bene trovato " - "Si ce n'est pas vrai, c'est au moins bien trouvé. "

"Monseigneur Provencher et son clergé séculier!» Me serais-je éloigné de mon sujet? En partie seulement, puisqu'il s'agissait de rétablir un peu plus de justesse sur l'esprit d'accueil de monseigneur Provencher vis-à-vis de ses prêtres qui, comme lui, avaient tout quitté pour partager son idéal de missionnaire.

La mission de la rivière Rouge a été exceptionnellement difficile. Il n'y a pas de voix plus autorisée que monseigneur Taché lui-même pour en parler. Dans son rapport à l'Association de la Propagation de la Foi de Lyon en $1888^{41}$, il décrit en particulier les cinq principales difficultés, et y inclut le commentaire suivant: Il faut en avoir fait l'expérience pour le savoir. Ces difficultés, qui conditionnent la vie des prêtres et qui doivent entrer en ligne de compte dans l'évaluation de leur apostolat, sont les suivantes:

1 - les voyages, 2 - la pauvreté, 3 - lisolement, 4 - lignorance des langues, 5 - l'inquiétude du succès.

37 Monice, Histoire de l'Eglise de COuest, I, p. 280. Il cite en note les paroles a de Provencher > prises dans Dugas, loc. cit.

38 Le père Antonio Champagne, Les La Vérendrye et le Poste de lOuest, Québec, Les Presses de l'Université Laval, 1968, Appen. II, p. 523: a François La Vérendrye et la fin d'un grand nom: 1769-1825 „.

39 Monseigneur Provencher et son Temps, Winnipeg, 1935, p. 208-209.

40 Les Missions Catholiques dans l'Ouest Canadien, Ottawa, 1949, p. 72.

41 Ce rapport a été republié dans Les Cloches de Saint-Boniface en 1915 (voir pages 109 et suivantes au cours de l'année). La mort de monseigneur Langevin, et les articles que l'on a publiés à ce sujet ont empêché le directeur des Cloches de publier tout le rapport de monseigneur Taché à la suite. 
C'est tout le rapport qu'il faudrait citer. Il est véritablement a Les Actes des Apôtres de l'Église dans le Nord-Ouest ». Quand on pense au fait qu'un des grands problèmes du clergé actuel semble être celui de "se situer ", on admettra sans peine qu'on en est à une période de décadence psychologique en comparaison avec les soixante-dix premières années de vie missionnaire dans l'Ouest.

J'ai pris la peine de tabuler les années de service du clergé de monseigneur Provencher, en comptant toute la durée de sa vie missionnaire, même si elle va au-delà de l'année 1853 , année de la mort de monseigneur Provencher ${ }^{42}$. Elles en disent long sur la persévérance de ces apôtres, malgré leurs fréquents remplacements.

Je les nommerai donc ici, non par ordre chronologique au service de la mission de la rivière Rouge, mais progressivement, selon le nombre de leurs années de service:

Blanchet, 1 mois; Demers, 1 an; Edge et Sauvé, 2 ans chacun; Darveau, 3 ans; Dumoulin, 5 ans; Poiré, 6 ans; Destroismaisons, 7 ans; Mayrand, 7 ans; Boucher, 8 ans; Harper, 9 ans; Laflèche et Bourassa, 12 ans chacun; Belcourt, 16 ans à Saint-Boniface et 12 ans à Pembina, pour un total de 28 ans; monseigneur Provencher, 35 ans; monseigneur Taché, 49 ans; l'abbé Thibault, 55 ans; et le père Lacombe, 64 ans, en plus de 2 ans à Pembina, en 1850.

De ces collaborateurs du temps de monseigneur Provencher, quelques-uns devinrent archevêques, soit monseigneur Blanchet et monseigneur Taché, d'autres évêques, soit monseigneur Demers, monseigneur Laflèche, monseigneur Faraud. Monseigneur Grandin n'arriva à SaintBoniface qu'un an après la mort de monseigneur Provencher.

Quand il fut question d'ériger Saint-Boniface en province ecclésiastique la première fois au temps de monseigneur Provencher, projet que celui-ci trouvait prématuré, il fut alors question de Belcourt à Pembina, de Laflèche à la Baie d'Hudson, et de Thibault à Edmonton. Monseigneur disait de Laflèche et Thibault qu'ils " avaient vu la mitre de près ». L'abbé Thibault fut vicaire général sous monseigneur Provencher, et l'abbé Belcourt, passé au diocèse de Dubucque puis de Saint-Paul, Minnesota, devint vicaire général de monseigneur Cretin, en $1852^{43}$.

Monseigneur Provencher a aimé ses prêtres, mais, vu la difficulté des missions, ceux-ci s'usaient vite à la tâche, puisque le ministère offrait peu de consolations. Les Saulteux ont été extrêmement lents à se convertir et, jusqu'à la fin de sa vie, monseigneur Provencher voyait encore des sauvages païens à proximité de sa cathédrale. Il avait un esprit de

42 J'ai utilisé pour cela les renseignements déjà tabulés au Régistre des Actes Episcopaux, AASB, II. Je n'ai pas fini de les compléter, malheureusement.

43 Voir lettre de l'abbé Albert Lacombe, in Les Cloches, 1917, p. 60. 
foi en la Providence à la mesure des épreuves qu'il eut à rencontrer. Peu de fois il a laissé paraître dans ses lettres la lassitude qu'il éprouvait certainement à avoir à attendre, simplement attendre. Il attendait la réponse à ses lettres qui ne venait qu'une fois l'an habituellement. Il attendait les décisions de la Compagnie de la Baie d'Hudson qui décidait des passages à accorder. Il attendait les résultats de son enseignement, mais pas une seule vocation ne s'épanouit au pays pendant son épiscopat. Il attendait les aumônes pour assurer à ses missionnaires le strict nécessaire, et les douze premières années, jusqu'en 1830, ces aumônes étaient presque illusoires. Cette mission de la rivière Rouge n'avait pas de sens, disait-on. Il manquait de ressources et il manquait de prêtres; et les conditions du ministère étaient loin d'être attrayantes. Il fallait persévérer malgré tout, et c'est là le plus grand succès de monseigneur Provencher. Par sa présence pendant trente-cinq ans à la rivière Rouge, lui-même soumis à la juridiction de l'archevêque de Québec de qui il dépendait en tout, il a tout de même réussi à coordonner l'effort missionnaire à la pleine mesure de ses moyens.

Quand il s'est agi de faire nommer un évêque pour le territoire de la Colombie, puisant dans sa propre expérience il disait:

Si cette mission prend des accroissements, il faudra songer à mettre un évêque à sa tête. Mon humble opinion est qu'il devra être indépendant. Cette mission que le diocèse de Québec aura la gloire d'avoir commencé malgré son grand éloignement ne pourra pas être fournie de sujets par lui. Si on peut introduire des prêtres tirés des corporations de France ce sera un très grand avantage pour le pays.

[...] J'insiste surtout sur l'indépendance de l'évêque qui pourra être nommé par la suite. Soyez bien persuadé qu'à une si grande distance et avec des communications difficiles, les progrès de la religion seront considérablement retardés, outre que les prêtres qui ne croient pas dépendre que par un fil de l'évêque qui n'a que des travaux pénibles à leur donner et qu'ils ne considèrent pas comme leur premier supérieur, mettront toujours légèrement la main à la charrue et regarderont souvent du côté d'où ils peuvent attendre un meilleur sort; de là la désaffection, etc. J'ai l'expérience et cependant je resterai comme je suis $\mathbf{4 4}$.

Ne pouvant faire justice aux collaborateurs de monseigneur Provencher par une brève notice historique sur chacun d'eux, je me bornerai à vous offrir un tableau qui rassemble bon nombre de détails historiques principaux. Je l'ajoute en annexe.

$\mathrm{Au}$ physique, monseigneur Provencher pouvait bien passer pour un géant dans les plaines de l'Ouest. Il était grand, six pieds et quatre pouces, exactement comme son cinquième successeur. En corpulence, il

44 Lettre Provencher-Signay, Rivière Rouge, 25 juin 1840, BSHSB, III. p. 184-191. Cette lettre serait à citer en entier. Elle est remplie de détails et d'informations sur les missionnaires de l'Ouest, les échanges de correspondance en France, à Saint-Louis, avec la Compagnie de la Baie d'Hudson, et des avis sur la bonne marche de la mission. 
ressemblait à votre conférencier. Dans une lettre il se prend à dire à son évêque:

Ma santé se conserve bonne, quoique je ne ressente aucune altération dans mon tempérament; $j$ 'ai cependant considérablement maigri. Le menton superflu est fondu, ainsi que le gros ventre; je n'en suis point aftligé, je n'en serai que mieux ${ }^{45}$.

Et moi de conclure: Heureux temps, où un prêtre pouvait maigrir honnêtement en accomplissant son devoir d'état et où l'évêque n'avait pas à lui dire: "Faudrait suivre une diète."

David Roy, prêtre,

Curé de Saint-Jean-Baptiste, Manitoba.

45 Lettre Provencher-Plessis, Fort Daer ou Pembina, 15 janvier 1819, BSHSB, III, p. 35 . 
MONSEIGNEUR PROVENCHER ET SON CLERGÉ SÉCULIER

\begin{tabular}{|c|c|c|c|c|c|}
\hline Nom & Naissance & Ordination & Arrivée-Départ & Qu'advint-il? & Décès \\
\hline 1. J.-Norbert Provencher & $\begin{array}{l}12 \text { février } 1787 \\
\text { Nicolet }\end{array}$ & $\begin{array}{l}21 \text { décembre } 1811 \\
\text { Québec }\end{array}$ & $\begin{array}{l}16 \text { juillet } 1818 \\
\text { Saint-Boniface }\end{array}$ & $\begin{array}{l}\text { Ev., } 1^{\text {er }} \text { février } 1820 \\
\text { C., } 12 \text { mai } 1822\end{array}$ & $\begin{array}{l}6 \text { juin } 1853 \\
\text { Saint-Boniface }\end{array}$ \\
\hline 2. Sévère Dumoulin & $\begin{array}{l}5 \text { décembre } 1793 \\
\text { Sainte-Anne, Montréal }\end{array}$ & 23 février 1817 & $\begin{array}{l}16 \text { juillet } 1818 \\
16 \text { juillet } 1823\end{array}$ & Curé de Yamachiche & $\begin{array}{l}27 \text { juillet } 1853 \\
\text { Yamachiche }\end{array}$ \\
\hline $\begin{array}{l}\text { 3. Guillaume Edge } \\
\text { ou William }\end{array}$ & $\begin{array}{l}1^{\text {er }} \text { décembre } 1792 \\
\text { Montréal }\end{array}$ & N'a pas persévéré & $\begin{array}{l}16 \text { juillet } 1818 \\
16 \text { août } 1820\end{array}$ & & \\
\hline $\begin{array}{l}\text { 4. Thomas-Ferruce Pic, } \\
\text { dit Destroismaisons }\end{array}$ & $\begin{array}{l}12 \text { janvier } 1796 \\
\text { Saint-Pierre, R. du Sud }\end{array}$ & 17 octobre 1819 & $\begin{array}{l}12 \text { août } 1820 \\
22 \text { juin } 1827 \\
\end{array}$ & & 5 avril 1866 \\
\hline $\begin{array}{l}\text { 5. Joseph Sauvé } \\
\text { ou Sauvez }\end{array}$ & $\begin{array}{l}8 \text { décembre } 1784 \\
\text { Vaudreuil }\end{array}$ & N'a pas persévéré & $\begin{array}{l}12 \text { août } 1820 \\
12 \text { août } 1822 \\
\end{array}$ & & \\
\hline 6. Jean Harper & $\begin{array}{l}6 \text { (18) septembre } 1801 \\
\text { Québec }\end{array}$ & $\begin{array}{l}1^{\text {er }} \text { novembre } 1824 \\
\text { Saint-Boniface } \\
\end{array}$ & $\begin{array}{r}7 \text { août } 1822 \\
23 \text { juillet } 1831 \\
\end{array}$ & $\begin{array}{l}\text { Curé de } \\
\text { Saint-Grégoire, P.Q. }\end{array}$ & $\begin{array}{l}30 \text { juillet } 1869 \\
\text { Saint-Grégoire, P.Q. }\end{array}$ \\
\hline 7. François Boucher & $\begin{array}{l}12 \text { mars } 1803 \\
\text { Saint-François, Beauce }\end{array}$ & $\begin{array}{l}16 \text { août } 1829 \\
\text { Saint-Boniface }\end{array}$ & $\begin{array}{l}19 \text { juin } 1827 \\
24 \text { juillet } 1833\end{array}$ & & \\
\hline $\begin{array}{l}\text { 8. Georges-Antoine } \\
\text { Belcourt }\end{array}$ & $\begin{array}{l}22 \text { avril } 1803 \\
\text { Baie du Fèbvre }\end{array}$ & $\begin{array}{l}10 \text { mars } 1827 \\
\text { Nicolet }\end{array}$ & $\begin{array}{l}17 \text { juin } 1831 \\
26 \text { octobre } 1847\end{array}$ & $\begin{array}{l}\text { Pembina, } 9 \text { juin } 1848 \\
\text { D. } 15 \text { mars } 1859\end{array}$ & $\begin{array}{l}31 \text { mai } 1874 \\
\text { S. Maméramcook, N.-B. }\end{array}$ \\
\hline $\begin{array}{l}\text { 9. Charles-Edouard } \\
\text { Poiré } \\
\end{array}$ & $\begin{array}{l}4 \text { août } 1810 \\
\text { Pointe-Lévis }\end{array}$ & $\begin{array}{l}17 \text { février } 1833 \\
\text { Saint-Boniface } \\
\end{array}$ & $\begin{array}{l}26 \text { juin } 1832 \\
11 \text { août } 1838 \\
\end{array}$ & V.G. de Québec & \\
\hline $\begin{array}{l}\text { 10. Jean-Baptiste } \\
\text { Thibault }\end{array}$ & $\begin{array}{l}\text { 14 décembre } 1810 \\
\text { Pointe-Lévis }\end{array}$ & $\begin{array}{l}8 \text { septembre } 1833 \\
\text { Saint-Boniface }\end{array}$ & $\begin{array}{r}\text { juin } 1833 \\
1888 \\
\end{array}$ & V.G. de $\mathrm{M}^{\mathrm{gr}}$ Provencher & \\
\hline 11. Modeste Demers & $\begin{array}{l}11 \text { octobre } 1809 \\
\text { Saint-Nicolas }\end{array}$ & $\begin{array}{l}7 \text { février } 1836 \\
\text { Québec }\end{array}$ & $\begin{array}{l}14 \text { juin } 1837 \\
10 \text { juillet } 1838\end{array}$ & $\begin{array}{l}\text { Evêque de Victoria } \\
\text { C., } 30 \text { novembre } 1847\end{array}$ & $\begin{array}{l}\text { juillet } 1869 \\
\text { Victoria, B.C. }\end{array}$ \\
\hline $\begin{array}{l}\text { 12. Joseph-Arsène } \\
\text { Mayrand }\end{array}$ & $\begin{array}{l}3 \text { mai } 1811 \\
\text { Deschambault }\end{array}$ & $\begin{array}{l}6 \text { avril } 1838 \\
\text { Québec }\end{array}$ & $\begin{array}{l}21 \text { juin } 1838 \\
29 \text { août } 184.5 \\
\end{array}$ & & $\begin{array}{l}24 \text { décembre } 1895 \\
\text { Province de Québec }\end{array}$ \\
\hline $\begin{array}{l}\text { 13. François-Norbert } \\
\text { Blanchet }\end{array}$ & $\begin{array}{l}30 \text { septembre } 1795 \\
\text { Saint-Pierre, R. du Sud }\end{array}$ & $\begin{array}{l}18 \text { juillet } 1819 \\
\text { Québec }\end{array}$ & $\begin{array}{r}5 \text { juin } 1838 \\
10 \text { juillet } 1838 \\
\end{array}$ & $\begin{array}{l}\text { Archevêque de Portland } \\
\text { C., } 25 \text { juillet } 1845 \\
\end{array}$ & $\begin{array}{l}188 ?, \\
\text { Portland, Orégon }\end{array}$ \\
\hline $\begin{array}{l}\text { 14. Jean-Edouard } \\
\text { Darveau } \\
\end{array}$ & $\begin{array}{l}17 \text { mars } 1816 \\
\text { Québec }\end{array}$ & $\begin{array}{l}21 \text { février } 1841 \\
\text { Québec }\end{array}$ & 10 juin 1841 & $\begin{array}{l}\text { Massacré par les sauvages } \\
\text { à la Baie des Canards }\end{array}$ & $\begin{array}{l}4 \text { juin } 1844 \\
\text { Lac Manitoba } \\
\end{array}$ \\
\hline $\begin{array}{l}\text { 15. Louis-François } \\
\text { Laflèche } \\
\end{array}$ & $\begin{array}{l}4 \text { septembre } 1818 \\
\text { Ste-Anne-de-la-Pérade }\end{array}$ & $\begin{array}{l}7 \text { janvier } 1844 \\
\text { Nicolet } \\
\end{array}$ & $\begin{array}{r}21 \text { juin } 1844 \\
1856 \\
\end{array}$ & $\begin{array}{l}\text { Evêque de Trois-Rivières } \\
\text { C., } 1^{\text {er }} \text { novembre } 1866 \\
\end{array}$ & $\begin{array}{l}14 \text { juillet } 1896 \\
\text { Trois-Rivières, P.Q. }\end{array}$ \\
\hline 16. Albert Lacombe & $\begin{array}{l}28 \text { février } 1827 \\
\text { Saint-Sulpice }\end{array}$ & $\begin{array}{l}13 \text { juin } 1849 \\
\text { Saint-Hyacinthe }\end{array}$ & $\begin{array}{l}\text { Pembina, } 7 \text { no- } \\
\text { vembre } 1850 \\
\text { Saint-Boniface, } \\
27 \text { juin } 1852 \\
\end{array}$ & & $\begin{array}{l}12 \text { décembre } 1916 \\
\text { Midnapore, Alberta }\end{array}$ \\
\hline
\end{tabular}

